\section{Taube Finger nach der Chemotherapie}

\begin{abstract}
Bei einer Reihe von Chemotherapieregimes kommt es zur chemotherapieinduzierten peripheren Neuropathie (CIPN). Aber wie groß ist das Risiko konkret? Wie lange dauert die CIPN an? Und wie sehr leiden die Patienten? Eine prospektive Studie gibt Antworten.
\end{abstract}

$\mathrm{D}$ a Krebspatienten häufiger und länger überleben, können und müssen Onkologen sich mehr um Nebenwirkungen und Langzeitfolgen der Krebsbehandlungen kümmern. $\mathrm{Zu}$ den wenig erforschten Folgen gehört die CIPN. Sie kann sehr belastend sein, da sie mit Taubheitsgefühlen und manchmal auch Schmerzen einhergeht. Besonders hoch ist das Risiko nach Therapie mit Platinderivaten, Vincaalkaloiden und Taxanen. Um das Ausmaß der CIPN und die dadurch auftretende Belastung genauer zu erfassen, untersuchten portugiesische Kollgen 296 Brustkrebspatientinnen im Rahmen einer prospektiven Studie.
Frauen, bei denen eine CIPN festgestellt wurde, wurden sechs Monate nach der Diagnose noch einmal evaluiert. Die kumulative Inzidenz für eine CIPN im ersten Jahr nach Diagnose betrug 28,7\% $(\mathrm{n}=85)$. Unter Patientinnen, die Docetaxel bekamen, betrug die Inzidenz 38,0\%. Im Mittel begann die CIPN 107 Tage nach Start der Chemotherapie. Zu Beginn hatten die meisten Patientinnen sensible Störungen leichteren Grads (12 ), nur bei $18,8 \%$ wurden motorische Störungen (vom Grad 1-3) festgestellt. 5 Patientinnen litten unter einer schmerzhaften CIPN. Eine Dosisreduktion war aber nur bei einer Patientin nötig. Bei mehr als $80 \%$ der Betroffenen hielten die Symptome mindestens 6 Monate an.

Für kumulative Docetaxel-Dosen von $<300 \mathrm{mg} / \mathrm{m}^{2}$ lag das relative Risiko bei 6,96, für Dosen $>300 \mathrm{mg} / \mathrm{m}^{2}$ bei 13,32. Alkoholkonsum und Diabetes waren nicht signifikant mit einer CIPN assoziiert. Es zeigten sich keine signifikanten Unterschiede im Outcome von betroffenen und nicht betroffenen Patientinnen.

Fazit: Eine CIPN war unter den hier beobachteten Patientinnen mit Brustkrebs im Frühstadium häufig. Sie war stark assoziiert mit Docetaxel-basierten Regimes und dosisabhängig. Die Symptome hielten bei den meisten Patientinnen mindestens 6 Monate an, aber ihre Schwere war gering.

Christina Berndt

Pereira S et al. Chemotherapy-induced peripheral neuropathy after neoadjuvant or adjuvant treatment of breast cancer: a retrospective cohort study. Support Care Cancer. 2016;24(4): 1571-81.

\title{
Die letzte Lebensphase erkennen
}

\begin{abstract}
Während der letzten Lebensmonate verschlechtern sich bei Patienten mit fortgeschrittener Krebserkrankung physische Funktionen und psychische Befindlichkeit oft stark. Wie hängt das mit der Überlebenszeit zusammen?
\end{abstract}

$B^{\mathrm{e}}$ ei Patienten mit fortgeschrittenem Krebsleiden gibt es typischerweise eine relativ lange Phase stabiler körperlicher Funktionen, gefolgt von rascher Verschlechterung in den letzten Lebensmonaten. Oft müssen die Patienten

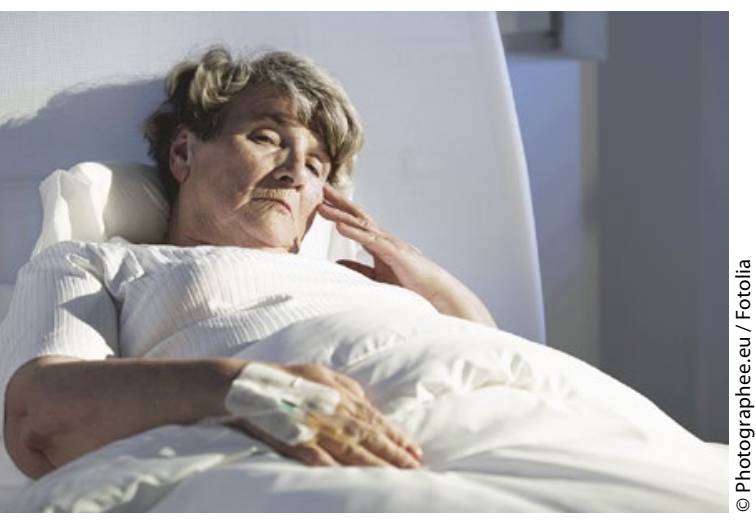

Wie stark die Depressivität ist, scheint mit dem Überleben zu korrelieren. dann hospitalisiert werden und benötigen eine kostenintensive Betreuung. Rechtzeitig zu bemerken, dass die letzte Lebensphase eingetreten ist, kann bei wichtigen Entscheidungen wie Therapien und Hospizeinweisungen helfen.

An der vorliegenden Studie nahmen 472 Patienten mit unheilbarer Krebserkrankung teil (mittleres Alter 58,9 Jahre, $56,6 \%$ Frauen). Binnen 18 Monaten wurden mehrfach Daten zu Angststörungen, Depression, Fatigue, Schmerzen und physischen Funktionen erhoben, daneben Befunde zu krebsspezifischen Symptomen. 203 Patienten $(43 \%)$ verstarben während des Follow-up.

Grundsätzlich waren die Scores für Angstzustände, Depression, Fatigue und Schmerzen höher als in der Allgemeinbevölkerung, die für körperliche Funktion niedriger. Die Scores für Depression, Fatigue, Schmerz und physische Funktionen waren hochsignifikante
Prädiktoren für das Überleben ( $\mathrm{p}<0,01)$. Große Unterschiede in der Überlebenswahrscheinlichkeit nach 6 und $12 \mathrm{Mo}$ naten zeigten sich zwischen Patienten mit Scores unterhalb der 25. und oberhalb der 75. Perzentile für Fatigue, Schmerzen und körperliche Funktionsfähigkeit (mit absoluten Differenzen von $10-18 \%$ ). Bei Patienten mit guter physischer Funktion $(\geq 75$. Perzentile) beispielsweise lag die Wahrscheinlichkeit, nach 6 bzw. 12 Monaten noch zu leben, bei 0,99 bzw. 0,89 , bei Patienten $\leq 25$. Perzentile bei 0,89 bzw. 0,72. Bei Patienten mit Schmerzen waren es entsprechend Wahrscheinlichkeiten von 0,90 bzw. 0,73 ( $\geq 75$. Perzentile) gegenüber 0,98 bzw. 0,87 ( $\leq 25$. Perzentile).

Fazit: Mit geeigneten Scores kann man valide abschätzen, wann ein Krebspatient in die letzte Lebensphase eintritt. Würden rechtzeitig adäquate Therapien eingeleitet, könnten zudem Beschwerden wie Fatigue und Schmerzen gelindert werden.

Kathrin von Kieseritzky

Stukenborg GJ et al. Longitudinal patterns of cancer patient reported outcomes in end of life care predict survival. Support Care Cancer. 2016; 24(5):2217-24 